\title{
Investigation of the Lipid Changes That Occur in Hypertrophic Muscle due to Fish Protein-feeding Using Mass Spectrometry Imaging
}

\author{
Mizuki Morisasa ${ }^{1}$, Naoko Goto-Inoue ${ }^{1 *}$, Tomohiko Sato ${ }^{1}$, Kazumasa Machida ${ }^{1}$, \\ Mina Fujitani ${ }^{2}$, Taro Kishida ${ }^{2}$, Kenji Uchida ${ }^{3}$, and Tsukasa Mori ${ }^{1}$ \\ ${ }^{1}$ Department of Marine Science and Resources, College of Bioresource Sciences, Nihon University, 1866 Kameino, Fujisawa, Kanagawa \\ 252-0880, JAPAN \\ ${ }^{2}$ Laboratory of Nutrition Science, Division of Applied Bioscience, Graduate School of Agriculture, Ehime University, Matsuyama, Ehime \\ 790-8566, JAPAN \\ ${ }^{3}$ Functional Ingredient Research Section, Food Function R\&D Center, Nippon Suisan Kaisha, Ltd., Tokyo 105-8676, JAPAN
}

\begin{abstract}
Alaska pollack protein (APP) was previously shown to reduce serum triacylglycerol and the atherogenic index and significantly increase gastrocnemius muscle mass in rats. To determine which myofibers are involved in this observed hypertrophy, we stained the gastrocnemius muscle with fast and slow fiber-specific antibodies and measured the muscle fiber diameter. We observed muscle hypertrophy in both the fast and slow fibers of APP-fed rats. Although muscle hypertrophy leads to drastic lipid changes, the amount of lipids did not differ significantly between casein-fed and APP-fed rats. To determine the lipid changes at the molecular species level and their localization, we performed matrix-assisted laser desorption/ ionization mass spectrometry imaging to visualize lipids in the gastrocnemius muscles. We determined that lipid molecules were significantly changed due to APP feeding. Thus, APP feeding changes muscle lipid metabolism, and these metabolic changes might be related to hypertrophy.
\end{abstract}

Key words: lipid, imaging, muscle, hypertrophy

\section{Introduction}

Muscle condition has important effects on human health. Sarcopenia syndrome leads to decreased skeletal muscle mass and strength, resulting in decreased quality of life. Muscle atrophy is caused by a reduction in protein synthesis, whereas muscle hypertrophy is induced by the synthesis of muscle protein. Therefore, promoting protein synthesis prevents sarcopenia. Several studies have suggested that nutritional supplements, exercise training, and dietary remedies might be effective treatments for sarcopenia ${ }^{1}$. In this study, we focused on a dietary remedy, fish protein, and studied the effect of feeding fish protein on skeletal muscle hypertrophy in rats with an aim to identify an effective means for muscle hypertrophy.

Fish protein is consumed worldwide and is frequently consumed in Japan. In particular, Alaska pollack (Theragra chalcogramma) is a material that is present in processed seafood, such as kamaboko and fish sausage. In a previous study, it was reported that the feeding of fish protein, spe- cifically Alaska pollack protein (APP), reduces serum triacylglycerol and the atherogenic index and significantly increases gastrocnemius muscle mass in rats ${ }^{2}$. It was also shown that skeletal muscle weight increased as a result of APP feeding over a short time period (approximately 7 days). In this study, we focused on the lipid dynamics in hypertrophic muscle to clarify the changes in lipids that occurred in association with the increased skeletal muscle mass, because in skeletal muscle, lipids play a fundamental role as an energy source, both in normal and diseased muscle, and have potential as molecular markers of muscle hypertrophy.

Muscle is composed of two types of muscle fibers, slow and fast, which have different metabolic characteristics, and these fibers are heterogeneously localized in the muscle. In gastrocnemius, we could divide it into three portions depends on the different ratio of these fibers ${ }^{3)}$. Muscle atrophy and hypertrophy have a tendency to occur predominantly in fast fibers. However, in previous studies

\footnotetext{
*Correspondence to: Naoko Goto-Inoue, Department of Marine Science and Resources, College of Bioresource Sciences, Nihon University, 1866 Kameino, Fujisawa, Kanagawa 252-0880, JAPAN

E-mail: inoue.naoko@ nihon-u.ac.jp

Accepted October 31, 2018 (received for review September 20, 2018)

Journal of Oleo Science ISSN 1345-8957 print / ISSN 1347-3352 online

http://www.jstage.jst.go.jp/browse/jos/ http://mc.manusriptcentral.com/jjocs
} 
using homogenized extracts, the localization information for metabolites was lost. Therefore, we attempted to identify which muscle fibers exhibit hypertrophic features and determine the lipid changes in the two fiber types by using matrix-assisted laser desorption/ionization mass spectrometry imaging (MS imaging). In addition, we attempted to identify molecular markers that may reveal the mechanism underlying muscle hypertrophy caused by APP feeding.

\section{Materials and Methods}

\subsection{Animals}

Five-week-old male Sprague-Dawley rats (SLC, Shizuoka, Japan)were housed in stainless steel wire mesh cages in a temperature-controlled room $\left(24 \pm 1^{\circ} \mathrm{C}\right)$ under a 12 -h lightdark cycle (dark phase: 15:00-3:00). At six weeks of age, the rats were assigned to one of two experimental groups: the casein diet group, which was fed the AIN-93 diet containing casein as the protein source, or the APP group, which was fed a diet containing APP instead of casein. The animals were housed separately for five days to acclimate them to the environment and throughout the 7-day feeding experiment with casein and APP feeding. After 7 days of feeding, the rats were sacrificed, and the gastrocnemius muscle was removed. This study was approved by the reviewing agency of Ehime University.

\subsection{Histochemical analysis}

Serial cross-sections of the muscles $(10-\mu \mathrm{m}$ thick) were cut using a cryostat (CM 1950; Leica Microsystems, Wetzlar, Germany), and the serial sections were mounted onto Matsunami Adhesive coated slides (Matsunami, Osaka, Japan) for histochemical staining or indium-tin-oxide-coated glass slides(Bruker Daltonics, Bremen, Germany) for MS imaging. For morphological observation, the sections were stained with hematoxylin and eosin $(\mathrm{H} \& \mathrm{E})$. To calculate the diameter of each myofiber, we measured the long radius of each fiber.

\subsection{Fluorescent immunohistochemical analysis}

To differentiate muscle fiber types, muscle sections were stained with antibodies against fiber type-specific antibodies. Tissue sections were fixed with $4 \%$ paraformaldehyde and incubated with primary antibodies against myosin skeletal, slow (GTX11083; Gene Tex, USA), troponin I fast (ab134838; Abcam, UK) and visualized using Alexa Fluor 488 and 568 fluorescence-conjugated secondary antibodies (Invitrogen). Fluorescence was detected with a wide field fluorescence microscope(BZ-9000; Keyence, Osaka, Japan).

\subsection{Lipid analysis by thin-layer chromatography (TLC)}

Total lipids were extracted from gastrocnemius muscle samples with chloroform: methanol $(2: 1, \mathrm{v} / \mathrm{v})$, and lipid fractions were extracted by the method of Bligh and Dyer as described previously ${ }^{4)}$. Equal amounts of the extracts were manually applied to silica gel 60 high-performance TLC plates (Merck, Darmstadt, Germany). The plates were developed with a solvent system consisting of methyl acetate/1-propanol/chloroform/methanol/0.25\% aqueous potassium chloride (25:25:25:10:9, v/v/v/v/v) for phospholipids, and a system composed of n-hexane/diethyl ether/ $\operatorname{acetic} \operatorname{acid}(80: 30: 1, \mathrm{v} / \mathrm{v} / \mathrm{v})$ for neutral lipids separation. The chromatograms were sprayed with primuline reagent, and the lipid bands were visualized under ultraviolet light. The relative densities of each lipid were quantitatively determined by ImageJ software(http://rsbweb.nih.gov/ij/). For TLC imaging, the lipids developed on the TLC plates were transferred to a polyvinyl difluoride membrane as described previously ${ }^{5}$.

\subsection{MS imaging}

MS imaging analyses were performed using a $\mathrm{TOF} / \mathrm{TOF}^{\mathrm{TM}}$ 5800 System (AB SCIEX). Analyses were performed in positive-ion mode for masses in the range of $\mathrm{m} / z 450-1000$, and in negative-ion mode for masses in the range of $\mathrm{m} / \mathrm{z}$ 200-1800. The matrices for ionization used $50 \mathrm{mg} / \mathrm{mL}$ 2,5-dihydroxybenzoic acid in methanol/water (8:2, v/v) for positive ion mode and $20 \mathrm{mg} / \mathrm{mL} 9$-aminoacrine in ethanol/ water $(8: 2, \mathrm{v} / \mathrm{v})$ for negative ion mode. The ion images were constructed using Datacube Explorer software ${ }^{6)}$. The MS parameters were set to obtain the highest sensitivity. Cryosections for MS imaging were prepared from three independent $\operatorname{rats}(n=3)$.

\subsection{Tandem mass spectrometric analyses}

Tandem mass spectrometric analysis (MS/MS) was performed directly on tissue sections of the skeletal muscle. The conditions for data acquisition (laser power and collision energy) were changed to obtain product-ion mass spectra with high intensity peaks and a high signal-to-noise ratio.

\subsection{Statistical analysis}

All data are expressed as mean \pm standard error of the mean (SEM). Statistical analyses were performed using Student's $t$ test by StatView 5.0 (SAS Institute, Tokyo, Japan). The level of significance was set at $p<0.05(*)$ or $p$ $<0.01(* *)$.

\section{Results}

Our previous report showed significantly increased volume in the gastrocnemius muscle of APP-fed rats compared to the volume of the muscle in control rats ${ }^{2}$. We prepared frozen tissue sections to confirm skeletal muscle hy- 
pertrophy by measuring muscle fiber diameter. Frozen sections were cut from the sagittal gastrocnemius muscles. Because of the difference in muscle fiber composition, we divided the muscle into three analytical regions, lateral head gastrocnemius of surface (LGasS), deep (LGasD) and medial head gastrocnemius of deep (MGasD). LGasS consists of fast fiber; LGasD and MGasD consists of fast and slow fiber, the ratio of slow fiber is greater in MGasD than LGasD. We first compared the muscle fiber diameter in casein-fed rats (Casein rats) and APP-fed rats (APP rats). As shown in Fig. 1A, the APP rats showed significantly larger muscle diameter in all regions (LGasS, LGasD, and MGasD). These data suggested that APP rats exhibited typical muscle hypertrophy. Next, we stained the muscle sections with antibodies against proteins specific for fast and slow fibers to confirm whether the hypertrophy was derived from fast or slow fibers. As shown in Fig. 1B, the diameters of both fiber types were significantly increased by approximately 1.2 -fold. In the LGasD portion of the muscle, the muscle fiber diameters in Casein rats were approximately $60 \mu \mathrm{m}$, whereas the median fiber diameter in APP rats was approximately $75 \mu \mathrm{m}$ for both the fast and slow fibers. In the MGasD, the muscle fiber diameter in Casein rats was approximately $55 \mu \mathrm{m}$, which was less than that in the LGasD. In contrast, the diameter in APP rats was approximately $60 \mu \mathrm{m}$ for fast fibers and $70 \mu \mathrm{m}$ for slow fibers. It is well known that the LGasS portion of the muscle is mostly composed of fast fibers, and our data also demonstrate that LGasS was predominantly stained with fast fiber-specific antibodies (data not shown). In short, our data show that muscle hypertrophy occurred in both fiber types.

To determine the lipidomic changes that occurred in association with muscle hypertrophy, we analyzed extracted lipids for the muscle tissues and evaluated them by using TLC. Extracted lipids were developed using two different solvent systems for polar lipid separation (Fig. 2A) and neutral lipid separation(Fig. 2B). In the polar lipid separation, phosphatidylethanolamine (PE) and phosphatidylcholine (PC) were mainly observed. We quantified the amount of $\mathrm{PE}$ and $\mathrm{PC}$ and confirmed that there was no significant difference in the levels between the Casein and APP rats. Triacylglycerol(TAG) was the main lipid in the neutral lipid separation, and the amount was nearly equal in the two groups of rats.

TLC analyses demonstrated that there was no significant change in the amount of major lipids such as PC, PE, and TAG. Therefore, we tried to detect lipid variation, as the lipids differed with fatty acid composition. We blotted lipids on the TLC plate to a polyvinyl difluoride membrane and performed MS imaging analyses of the PC band regions (Fig. 2C). As shown in the figure, we found several molecular ions in the PC bands. We selected three molecular species at $m / z 782.5,828.5$, and 856.6 and examined their ion images on the TLC plates. The molecular ion at $\mathrm{m} / z$ 782.5 was the highest intensity in PC bands. And the molecular ion at $\mathrm{m} / z 828.5$ and 856.6 were also detected in previous study $^{7)}$. Based on the previous report, we were able to assign these ions as $[\mathrm{M}+\mathrm{Na}]^{+} \mathrm{PC}(16: 0 / 18: 1),[\mathrm{M}+$
A
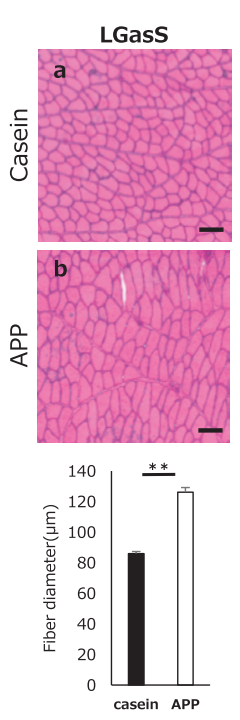
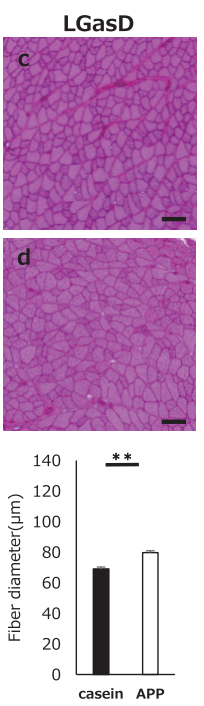
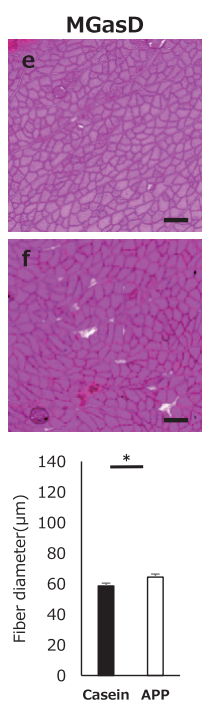

B
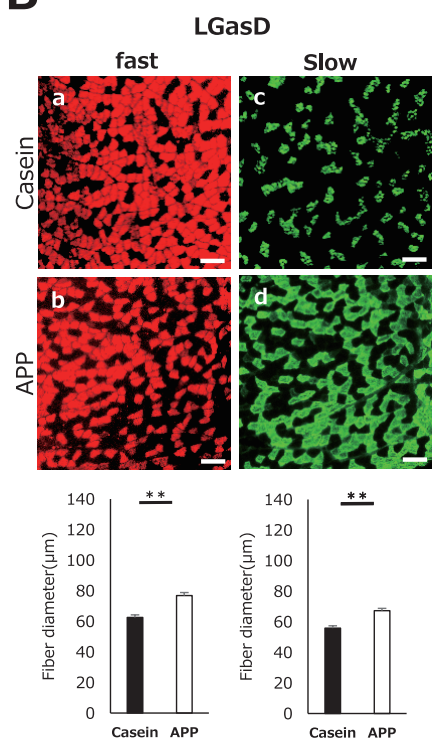
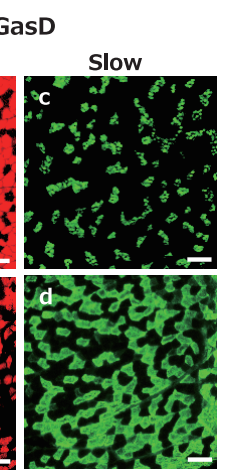
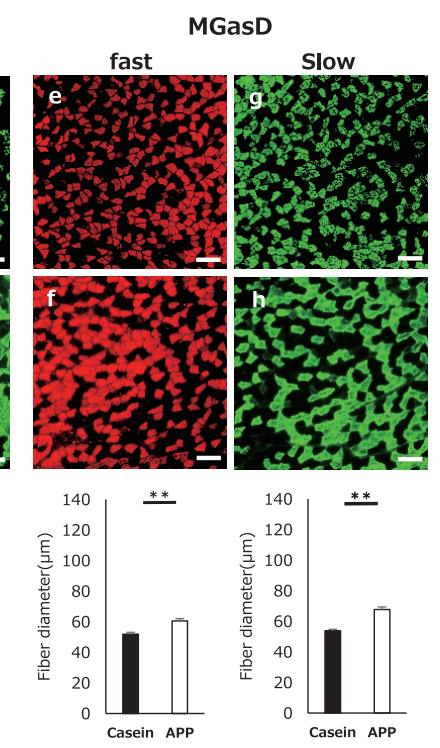

Fig. 1 The change in muscle fiber diameter caused by APP feeding. (A) H\&E stained cryosections of different portions of the gastrocnemius; lateral head gastrocnemius of surface (LGasS), deep (LGasD) and medial head gastrocnemius of deep (MGasD) from Casein and APP rats. (B) Immunofluorescence imaging of muscle sections stained with fast and slow fiber-specific antibodies. The diameter was calculated $(\mathrm{n}=100)$, and statistical analyses were performed. ${ }^{*} p<$ $0.05, * * p<0.01$. Scale bar: $125 \mu \mathrm{m}$. 

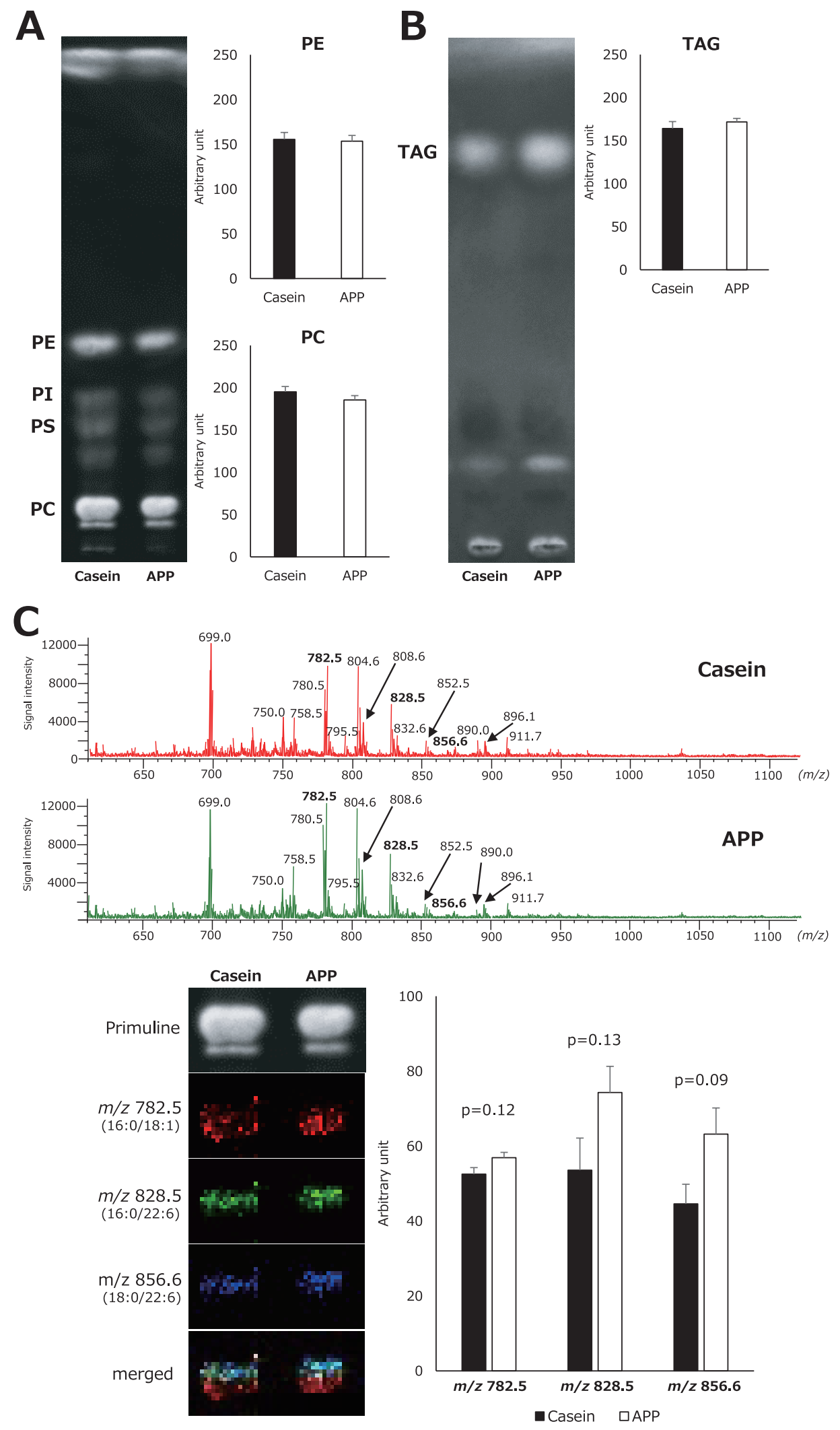

Fig. 2 Thin layer chromatogram of separated polar lipids (A) and neutral lipids (B). (C) Mass spectra of PC regions from a blotted PVDF membrane (upper), and the ion images at $\mathrm{m} / \mathrm{z} 782.5,828.5$, and 856.6 are shown. The bar graph shows the signal intensities of each ion. 

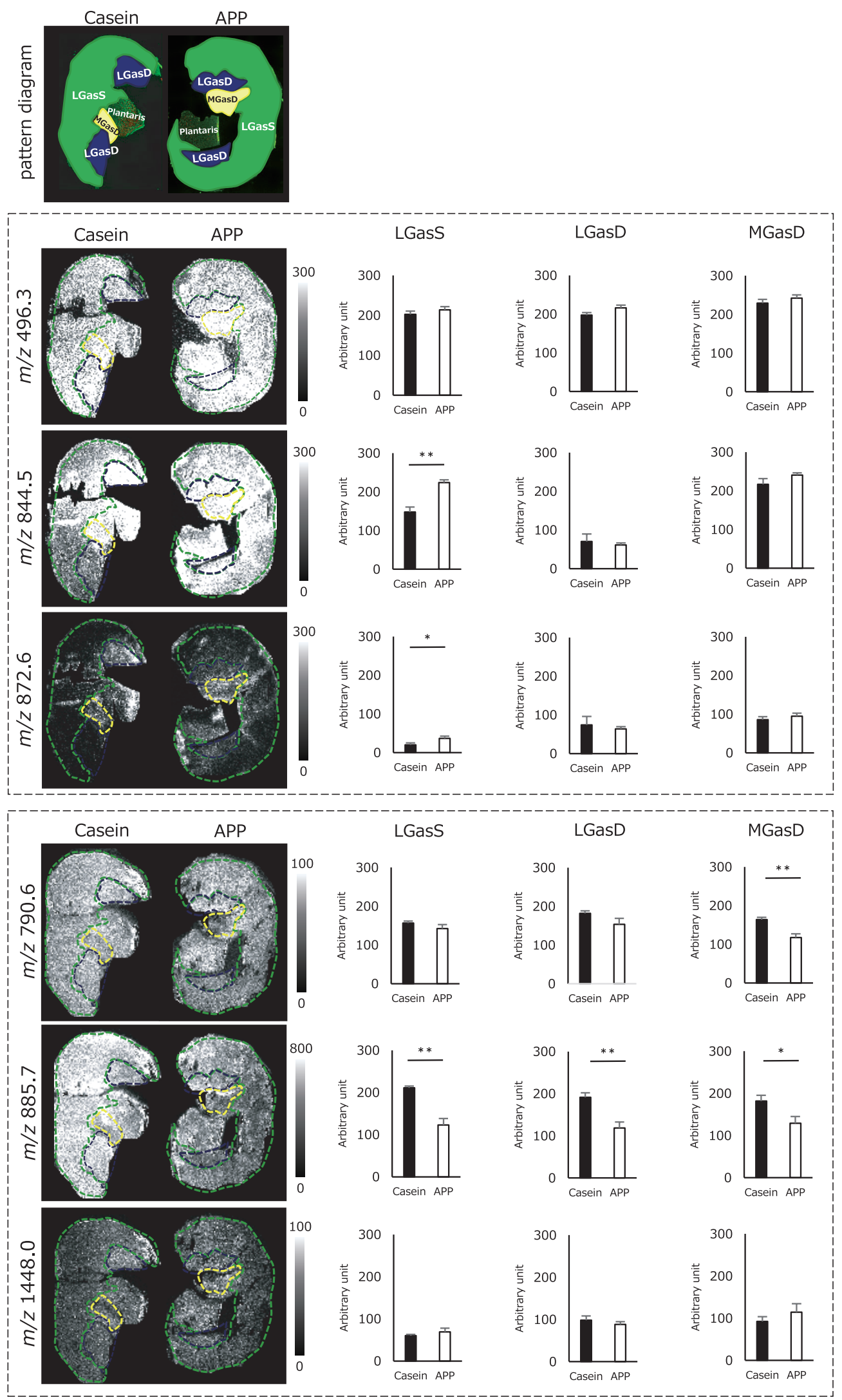

Fig. 3 MS imaging results in positive ion mode (upper) and negative ion mode(lower). The molecular distribution of the ions at $m / z 496.3,844.5,872.6,790.6,885.7$, and 1448.0 are shown. $* * p<0.01$. 
$\mathrm{Na}]^{+} \mathrm{PC}(16: 0 / 22: 6)$, and $[\mathrm{M}+\mathrm{Na}]^{+} \mathrm{PC}(18: 0 / 22: 6)^{8)}$. Statistical analyses were performed to compare the amount of each molecular species, and the ion image of PC (18:0/22:6) at $m / z 856.6$ was relatively higher in APP rats than in Casein rats $(p=0.09)$. These data suggested that the molecular species composition of PC might have changed in the APP rats when compared to the control rats. However, these analyses used homogenized whole gastrocnemius tissues, therefore any fiber-specific lipid changes were lost.

Next, we performed MS imaging of sagittal sections of the gastrocnemius muscle. From the immunofluorescence staining, we were able to distinguish the three different muscle parts. In the pattern diagram, the LGasS, LGasD, and MGasD regions are shown in green, blue, and yellow, respectively. We detected various ions, and representative ion images are shown in Fig. 3. The signal intensities were calculated, and statistical analyses were performed for the three regions. The ion at $m / z 496.3$ was strongly detected in gastrocnemius, and there was no significant difference in all regions of the gastrocnemius between the Casein and APP rats. The intensity of the molecular ion at $\mathrm{m} / z 844.5$ was highest in the MGasD. And the signals in the LGasS were significantly higher in APP rats than in Casein rats. The molecular ion at $m / z 872.6$ was highly detected in the LGasD and MGasD portions. Interestingly, in the LGasS, significantly higher intensities were detected in APP rats than in Casein rats.

The lower panel in Fig. 3 shows the ions detected in negative-ion mode. We detected three peaks at $\mathrm{m} / \approx 790.6$, 885.7, and 1448.0. Based on previous reports, the signal at $\mathrm{m} / \approx 790.6$ was assigned as $\mathrm{PE}^{9)}$. Because $\mathrm{PE}$ is a main component of the inner cell membrane, this signal was uniformly observed. The signal intensity in the MGasD was significantly lower in APP rats than Casein rats. The signal at $m / z 885.7$ was high in all regions, although the signal intensities in all regions were lower in APP rats than in Casein rats. The localization of the ion at $\mathrm{m} / \approx 1448.0$ mirrored that of cardiolipin, which is specific to mitochondria. The signal did not differ significantly between APP and Casein rats.

To confirm the molecular structure, we performed MS/
MS. Figure 4A shows the product-ion spectrum of the peak at $\mathrm{m} / \mathrm{z}$ 496.3. Fragment peaks were detected at $\mathrm{m} / \mathrm{z} 184.2$ $\left[\left(\mathrm{CH}_{3}\right)_{3} \mathrm{~N}\left(\mathrm{CH}_{2}\right)_{2} \mathrm{PO}_{4} \mathrm{H}+\mathrm{H}\right]^{+}$and $m / z 313.5(\mathrm{M}-183+\mathrm{H})^{+}$. The product-ion spectrum showed peaks at $\mathrm{m} / z 104.2$ $\left[\left(\mathrm{CH}_{3}\right)_{3} \mathrm{~N}\left(\mathrm{CH}_{2}\right)_{2} \mathrm{O}+\mathrm{H}\right]^{+}$and $m / z 125.1\left[\left(\mathrm{CH}_{2}\right)_{2} \mathrm{PO}_{4} \mathrm{H}+\mathrm{H}\right]^{+}$ from the headgroup. Based on the above results, the species at $m / z 496.3$ is protonated LysoPC (18:0). Figure 4B shows product-ion spectra of $\mathrm{m} / z$ 844.5. Fragment peaks were detected at $\mathrm{m} / \approx 184.1\left[\left(\mathrm{CH}_{3}\right)_{3} \mathrm{~N}\left(\mathrm{CH}_{2}\right)_{2} \mathrm{PO}_{4} \mathrm{H}+\right.$ $\mathrm{H}]^{+}, m / z 661.5(\mathrm{M}-183+\mathrm{K})^{+}$, and $m / z 785.6(\mathrm{M}-59+\mathrm{K})^{+}$, and the molecule was assigned as $[\mathrm{M}+\mathrm{K}]^{+} \mathrm{PC}(16: 0 / 22: 6)$. Figure $4 \mathrm{C}$ shows the product-ion spectra for the peak at $\mathrm{m} / \approx$ 872.6. Fragment peaks were detected at $\mathrm{m} / z 588.3$ $(\mathrm{M}-284+\mathrm{K})^{+}, \mathrm{m} / \mathrm{z} 689.6(\mathrm{M}-183+\mathrm{K})^{+}$, and $\mathrm{m} / \mathrm{z} 813.6$ $(\mathrm{M}-59+\mathrm{K})^{+}$, and it was assigned as $[\mathrm{M}+\mathrm{K}]^{+} \mathrm{PC}(18: 0 /$ 22:6).

\section{Discussion}

To confirm how muscle hypertrophy occurs in our animal model, we used MS imaging to perform a region-specific characterization of the lipids. We divided the gastrocnemius muscle region of interest into the LGasS, LGasD, and MGasD portions, in which the fiber compositions vary. The results showed that muscle hypertrophy occurred in both fiber types evenly everywhere.

We analyzed the lipids extracted from these muscles, including PC, PE, and TAG, by using TLC. However, there were no significant differences between the Casein and APP rats. We also investigated the molecular species of PC, which differ in their fatty acid chain length. The molecular ion of PC (18:0/22:6) at $m / z$ 856.6 showed a tendency to increase in the APP group, as shown by the TLC imaging ( $p$ $=0.09$ ). In our previous study, we demonstrated that $\mathrm{m} / \mathrm{z}$ 844.5 and $\mathrm{m} / \mathrm{z} 872.6$ were the main PC molecular ions in murine muscle. Specifically, $\mathrm{m} / z 844.5$ was detected throughout the muscle sections, whereas the ion at $\mathrm{m} / \mathrm{z}$ 872.6 was detected only in slow fibers ${ }^{10)}$. In our MS imaging results, the ion at $m / z 872.6$ was highly detected in the LGasD and MGasD portions, and this molecule was as-
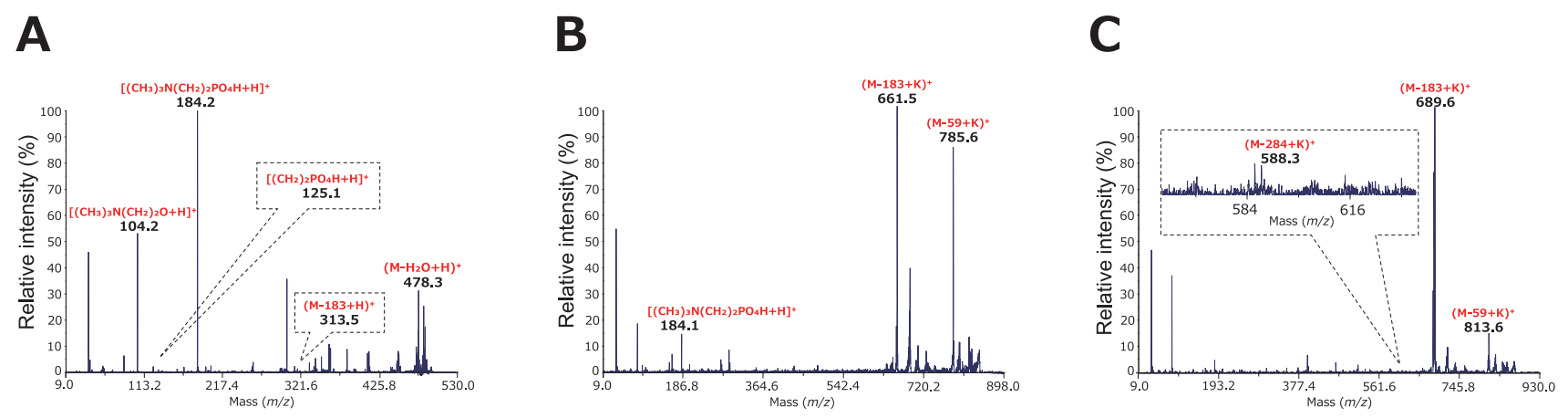

Fig. 4 Tandem mass spectra of precursor ions at $m / z 496.3$ (A), $m / z 844.5$ (B), and $m / z 872.6$ (C). 
signed as PC (18:0/22:6). The results of the TLC imaging and MS imaging showed a increased tendency of PC (18:0/22:6) in APP rats. Furthermore, PC (18:0/22:6), which is docosahexaenoic acid (DHA)-containing PC, was significantly increased in the LGasS where fast fiber types are predominant. Our previous report demonstrated that $\mathrm{PC}(18: 0 / 22: 6)$ is a slow fiber-specific lipid ${ }^{11)}$. These data suggest that APP feeding might convert the lipid composition of fast fibers to that of slow fibers. The accumulation of DHA-containing PC has positive effects on the prevention of obesity ${ }^{12)}$, and the amount of PC (18:0/22:6) was increased in a chronic training-induced model of muscle hypertrophy ${ }^{4)}$. Therefore, the increase of PC (18:0/22:6) due to APP feeding suggested that APP has effects on lipid metabolism. We hypothesized that PC (18:0/22:6) might be a useful molecular marker of APP feeding-induced hypertrophy in fast fiber. These localized differences may be lost in analyses of homogenized tissue. In the near future, we will attempt a more detailed muscle fiber typing (I, IIa, IIb, and IIx) to visualize the fiber compositions in all areas by MS imaging.

There was no significant difference between APP and Casein rats in the major ion at $m / z 790.6$, which was predicted to be PE (16:0/22:6), in both the TLC analysis and MS imaging of LGasS and LGasD. However, the signal intensity was significantly lower in APP rats than Casein rats in the MGasD. The molecular ion at $\mathrm{m} / z 885.7$ could be assigned as PI(18:0/20:4), a major molecular species of PI. However, the TLC analyses demonstrated that the total amount of PI was nearly equal in the APP and Casein rats, whereas the MS imaging results suggested that PI (18:0/20:4) was decreased in all portions. We realize that these differences rely on the muscle fiber composition, which varies depending on the region. Therefore, in the future, we will focus on these fiber-specific changes in greater detail at higher spatial resolution. The molecular ion at $m / z$ 1488.0, cardiolipin (18:2/18:2/18:2/18:2), was the most abundant molecular species, and therefore may be useful as a mitochondrial marker.

A previous report demonstrated that the intake of fish oils induced a muscle fiber shift ${ }^{13)}$. In the present study, we assessed the effect of fish protein intake and observed the hypertrophic features of the muscle. Based on our immunofluorescence results, the ratio of fast/slow fibers did not change. However, fiber-dependent lipid changes were observed by MS imaging. Finally, we demonstrated that DHAcontaining $\mathrm{PC}$ is a molecular marker that changed as a result of APP feeding in fast fiber.

\section{References}

1) Xia, Z.; Cholewa, J.M.; Zhao, Y.; Yang, Y.; Shang, H.; Jiang, H.; Su, Q.; Zanchi, N.E. A potential strategy for counteracting age-related sarcopenia: preliminary evidence of combined exercise training and leucine supplementation. Food Funct. 8, 4528-4538 (2017).

2) Mizushige, T.; Kawabata, F.; Uozumi, K.; Tsuji, T.; Kishida, T.; Ebihara, K. Fast-twitch muscle hypertrophy partly induces lipid accumulation inhibition with Alaska pollack protein intake in rats. Biomed. Res. 31, 347-352 (2010).

3) Sawano, S.; Komiya, Y.; Ichitsubo, R.; Ohkawa, Y.; Nakamura, M.; Tatsumi, R.; Ikeuchi, Y.; Mizunoya, W. A one-step immunostaining method to visualize rodent muscle fiber type within a single specimen. PLoS One 11, e0166080 (2016).

4) Goto-Inoue, N.; Yamada, K.; Inagaki, A.; Furuichi, Y.; Ogino, S.; Manabe, Y.; Setou, M.; Fujii, N.L. Lipidomics analysis revealed the phospholipid compositional changes in muscle by chronic exercise and high-fat diet. Sci. Rep. 3, 3267 (2013).

5) Goto-Inoue, N.; Hayasaka, T.; Taki, T.; Gonzalez, T.V.; Setou, M. A new lipidomics approach by thin-layer chromatography-blot-matrix-assisted laser desorption/ ionization imaging mass spectrometry for analyzing detailed patterns of phospholipid molecular species. $J$. Chromatogr. A 1216, 7096-7101(2009).

6) Klinkert, I.; Chughtai, K.; Ellis, S.R.; Heeren, R.M.A. Methods for full resolution data exploration and visualization for large 2D and 3D mass spectrometry imaging datasets. Int. J. Mass Spectrom. 362, 40-47 (2014).

7) Zaima, N.; Goto-Inoue, N.; Adachi, K.; Setou, M. Selective analysis of lipids by thin-layer chromatography blot matrix-assisted laser desorption/ionization imaging mass spectrometry. J. Oleo Sci. 60, 93-98(2011).

8) Hayasaka, T.; Goto-Inoue, N.; Zaima, N.; Kimura, Y., Setou, M. Organ-specific distributions of lysophosphatidylcholine and triacylglycerol in mouse embryo. Lipids 44, 837-848(2009).

9) Wang, J.; Wang, C.; Han, X. Enhanced coverage of lipid analysis and imaging by matrix-assisted laser desorption/ionization mass spectrometry via a strategy with an optimized mixture of matrices. Anal. Chim. Acta 1000, 155-162 (2018).

10) Goto-Inoue, N.; Manabe, Y.; Miyatake, S.; Ogino, S.; Morishita, A.; Hayasaka, T.; Masaki, N.; Setou, M.; Fujii, N.L. Visualization of dynamic change in contraction-induced lipid composition in mouse skeletal muscle by matrix-assisted laser desorption/ionization imaging mass spectrometry. Anal. Bioanal. Chem. 403, 1863-1871 (2012).

11) Senoo, N.; Miyoshi, N.; Goto-Inoue, N.; Minami, K.; Yoshimura, R.; Morita, A.; Sawada, N.; Matsuda, J.; Ogawa, Y.; Setou, M.; Kamei, Y.; Miura, S. PGC-1alpha-mediated changes in phospholipid profiles of exercisetrained skeletal muscle. J. Lipid Res. 56, 2286-2296 
(2015).

12) Cheng, K.K.; Akasaki, Y.; Lecommandeur, E.; Lindsay, R.T.; Murfitt, S.; Walsh, K.; Griffin, J.L. Metabolomic analysis of akt1-mediated muscle hypertrophy in models of diet-induced obesity and age-related fat accumulation. J. Proteome Res. 14, 342-352(2015).
13) Mizunoya, W.; Iwamoto, Y.; Shirouchi, B.; Sato, M.; Komiya, Y.; Razin, F.R.; Tatsumi, R.; Sato, Y.; Nakamura, M.; Ikeuchi, Y. Dietary fat influences the expression of contractile and metabolic genes in rat skeletal muscle. PLoS One 8, e80152(2013). 